\title{
CXCL14 facilitates the growth and metastasis of ovarian carcinoma cells via activation of the Wnt/ $\beta$-catenin signaling pathway
}

\author{
Li-Na Gao ${ }^{\dagger}$, Man Hao ${ }^{*}$, Xiao-Hui Liu ${ }^{\dagger}$, Li Zhang, Yan Dong, Yu-Fang Zhang and Xiao-Chun He
}

\begin{abstract}
Background: There is an urgent need to identify potential targets in anticancer therapy to improve the survival and prognosis of patients with ovarian cancer (OC). Herein, we investigated the functional significance of chemokine (C-X-C motif) ligand 14 (CXCL14) in OC cell growth and epithelial-mesenchymal transition (EMT).

Methods: qRT PCR and western blotting was used to detect CXCL14 mRNA level and protein expression, respectively. The functional mechanism of CXCL14 in OC was investigated by CCK-8, colony formation and transwell assays. The migration ability of OC cell was determined using wound healing. The protein expressions of CXCL14 and $\beta$-catenin in OC tissues were determined by immumohistochemical staining.

Results: We demonstrated that high levels of CXCL14 were associated with a worse prognosis in patients with OC. CXCL14 knockdown considerably restrained the growth, migration and invasion of OC cell in vitro. In contrast, ectopic CXCL14 overexpression yielded the opposite results. Investigations to determine the underlying molecular mechanisms revealed that the Wnt/ $\beta$-catenin signaling pathway is involved in CXCL14-facilitated OC cell invasiveness.
\end{abstract}

Conclusion: These data collectively demonstrate that CXCL14 contributes to OC cell growth and metastatic potential by regulating the $W n t / \beta$-catenin signaling pathway.

Keywords: CXCL14, Ovarian cancer, EMT, Wnt/ $\beta$-catenin

\section{Introduction}

Ovarian cancer $(\mathrm{OC})$ is the most lethal cancer among gynecologic malignancies [1]. Despite considerable improvements in surgery and chemoradiotherapy, survival rate remains deplorable owing to recurrences and metastases [2]. Recent clinical and experimental evidence demonstrates that epithelial-mesenchymal transition (EMT) is a critical event in the process of cancer cell metastasis [3]. EMT is a transdifferentiation process, in which epithelial cells undergo changes and demonstrate

\footnotetext{
*Correspondence: dchaomanhm@163.com

${ }^{\dagger}$ Li Na Gao, Man Hao and Xiao-Hui Liu contributed equally to this work.

The Second Obstetrics Department, Gansu Provincial Maternity and Child-care Hospital, No. 143 North Qilihe Street, Qilihe District, Lanzhou, Gansu, China
}

mesenchymal phenotypes, and is mainly characterized by the gain of the mesenchymal biomarker, N-cadherin, and the loss of the epithelial biomarker, E-cadherin [4].

CXCL14 is a key homeostatic chemokine that facilitates immune surveillance of skin epithelium through recruitment of various immune cell types, including natural killer cells, dendritic cells (DCs), and T cells [5]. A study has investigated the abnormal expression of CXCL14 and its biological functions in diverse carcinomas [6]. Furthermore, CXCL14 has been demonstrated to have both tumor-suppressive and pro-oncogenic roles. For example, CXCL14 exerts anticancer effects in head and neck cancer, colorectal carcinoma, and oral cancer $[7,8]$. Moreover, CXCL14 expression is downregulated in breast cancer, and CXCL14 overexpression inhibits cell growth and invasion in vitro and attenuates tumor original author(s) and the source, provide a link to the Creative Commons licence, and indicate if changes were made. The images or other third party material in this article are included in the article's Creative Commons licence, unless indicated otherwise in a credit line to the material. If material is not included in the article's Creative Commons licence and your intended use is not permitted by statutory regulation or exceeds the permitted use, you will need to obtain permission directly from the copyright holder. To view a copy of this licence, visit http://creativecommons.org/licenses/by/4.0/. The Creative Commons Public Domain Dedication waiver (http://creativecommons.org/publicdomain/zero/1.0/) applies to the data made available in this article, unless otherwise stated in a credit line to the data. 
growth and pulmonary metastasis of breast carcinoma cells in vivo [9]. However, CXCL14 expression is higher in glioblastoma tissues than in surrounding healthy tissues, and CXCL14 enhances the migration and proliferation of glioblastoma cells [10].

Earlier investigations have closely linked chemokines and their membrane receptors to the pathological EMT process [11]. For instance, Wang demonstrated that C-C motif chemokine-17 (CCL-17) was secreted from CXCL14-activated fibroblasts as a regulatory factor of the CXCL14-triggered breast carcinoma cell EMT and metastasis [12]. Additionally, CXCL14 was identified to function as a chemokine for the M2-type macrophage, which triggers EMT [13]. Upregulated CXCL14 expression is associated with poor overall survival of patients, as it promotes OC cell proliferation [14]. However, the role of CXCL14 in EMT and the metastasis of OC, as well as the molecular mechanism, has not been elucidated.

In this study, we evaluated the expression of CXCL14 in patients with $\mathrm{OC}$ and determined its correlation with the clinical outcomes of patients. Furthermore, we explored the functional significance of CXCL14 expression in OC cell invasion and EMT.

\section{Materials and methods}

\section{Cell culture}

The human ovarian surface epithelial cell line HOSEpiC and four ovarian carcinoma cell lines (OVCAR3, SKOV3, A2780, and CAOV3) were purchased from Nanjing KeyGen Biotech (Nanjing, China). Cells were cultured in DMEM (Thermo Fisher Scientific, Waltham, MA, USA) supplemented with $10 \% \mathrm{FBS}, 100 \mathrm{U} / \mathrm{ml}$ penicillin and $0.1 \mathrm{mg} / \mathrm{ml}$ streptomycin (Gibco, Carlsbad, CA, USA) at $37^{\circ} \mathrm{C}$ in an incubator with $5 \% \mathrm{CO}_{2}$. The inhibitor of Wnt/ $\beta$-catenin signaling, XAV939, was purchased from Selleck Chemicals (Shanghai, China).

\section{Tissue samples}

Fifty patients with ovarian cancers and 50 patients with benign epithelial ovarian tumor were obtained at operation in the Gansu Provincial Maternity and Child-care Hospital. OC tissues $(n=50)$ and non-tumor ovarian tissues $(\mathrm{n}=50$, normal ovarian tissues more than $5 \mathrm{~cm}$ away from the benign epithelial ovarian tumor's margin) were acquired from OC patients or patients with benign epithelial ovarian tumor, respectively [15]. Written informed consent was obtained from all the patients. Paraffinembedded tissues were cut into $4-\mu \mathrm{m}$-thick sections. The immunohistochemistry procedure to determine CXCL14 and $\beta$-catenin expression was performed as described previously $[16,17]$. To evaluate CXCL14 or $\beta$-catenin staining, its extent was scored by assigning a staining percentage to positive tumor cells $(0$, none; $1,<20 \%$ of positive staining cells; $2,20-50 \%$ of positive staining cells; $3,>50 \%$ of positive staining cells). Samples with scores of 0,1 , or 2 were defined as low expression, while cases with a score of 3 were defined as having high expression. The study was approved by the Medical Ethical Committee of Gansu Provincial Maternity and Child-care Hospital.

\section{Plasmid transfections}

CXCL14 shRNA (sh-CXCL14 \#1, sh-CXCL14 \#2) and shRNA negative control (sh-NC) were obtained from GeneCopoeia (Guangzhou, China). pcDNA3.1-CXCL14 (CXCL14-OE) and pcDNA3.1-empty vector (Vector) were purchased from GenePharma (Shanghai, China). Transfection or co-transfection was performed using the Lipofectamine 3000 kit (Thermo Fisher Scientific) according to the manufacturer's instructions.

RNA extraction and quantitative real-time PCR (qRT-PCR) RNA was extracted from cells using the TRIzol $^{\circledR}$ kit (Thermo Fisher Scientific). First-strand cDNA was obtained using the PrimeScript ${ }^{\circledR}$ RT Kit (Takara, Dalian, China). Then, qRT-PCR was conducted using a SYBR Green Real-time kit (TaKaRa) on an IQ5 real-time PCR system (Bio-Rad). The following primers were used: CXCL14 forward, 5'-CGCTACAGCGACGTGAAG AA- $3^{\prime}$ and reverse, $5^{\prime}$-GTTCCAGGCGTTGTACCAC-3'; $\beta$-catenin forward, 5'-ATGGAGCCGGACAGAAAA GC-3' and reverse, 5'-CTTGCCACTCAGGGAAGG A-3'; MMP-7 forward, 5'-GAGTGAGCTACAGTGGGA ACA- $3^{\prime}$ and reverse, 5'-CTATGACGCGGGAGTTTA ACAT-3'; Cyclin D1 forward, 5'-GCTGCGAAGTGG AAACCATC- $3^{\prime}$ and reverse, $5^{\prime}$-CCTCCTTCTGCACAC ATTTGAA-3'; Axin2 forward, 5'-TGACTCTCCTTC CAGATCCCA-3' and reverse, 5'-TGCCCACACTAG GCTGACA-3'; and GAPDH forward, 5'-TGGATTTGG ACGCATTGGTC- $3^{\prime}$ and reverse, $5^{\prime}$-TTTGCACTGGTA CGTGTTGAT-3'. Quantification was performed using the $2^{-\Delta \Delta \mathrm{Ct}}$ method. GAPDH was used as the internal control.

\section{Cell proliferation assay}

Cell proliferation was determined using a Cell Counting Kit-8 (CCK-8) kit (Dojindo, Tokyo, Japan). Cells were collected on days $1,2,3$, and 4 . After incubation with CCK-8 reagent for $2 \mathrm{~h}$, the absorbance (OD) was measured using a microplate reader (Bio-Tek, USA) at $450 \mathrm{~nm}$.

\section{Colony formation assay}

One thousand cells were maintained in 6-well plates for 2 weeks in an incubator at $37^{\circ} \mathrm{C}$. Then, the cell colonies were fixed using $4 \%$ polyoxymethylene and stained with $1 \%$ crystal violet (Beyotime, Nanjing, China). The number of clones with more than 50 cells was counted. 


\section{Migration assay}

Cells $\left(1 \times 10^{4}\right)$ were seeded into 6 -well plates overnight to form a complete monolayer. Then, a $200 \mu$ pipette tip was used to make wounds across the cell monolayer. After removing dead cells with PBS, cells were cultured with serum-free DMEM medium for $24 \mathrm{~h}$. Each wound was photographed at $0 \mathrm{~h}$ or $24 \mathrm{~h}$, and the wound healing rate was calculated using the following formula: migration rate $=(0 \mathrm{~h}$ scratch width $-24 \mathrm{~h}$ scratch width $) / 0 \mathrm{~h}$ scratch width) $\times 100 \%$.

\section{Invasion assay}

The upper basement membrane of an 8 - $\mu$ m pore transwell chamber (Corning, NY, USA) was pre-coated with $20 \mu \mathrm{g}$ Matrigel. The cell suspension $\left(200 \mu \mathrm{L}, 1 \times 10^{3}\right)$ was plated in the upper compartment, and the lower compartment was filled with DMEM $(600 \mu \mathrm{L})$ supplemented with $20 \%$ FBS. After $48 \mathrm{~h}$, the invading cells were fixed with formaldehyde and stained with $1 \%$ crystal violet. The number of invading cells in five random visual fields was counted using a microscope.

\section{Immunoblotting}

Total protein was extracted and separated using $10 \%$ SDS-PAGE. To obtain nuclear and cytoplasmic fractions, subcellular fractionation was performed as described previously [18]. After transfer onto PVDF membranes (Millipore, Braunschweig, Germany), the membranes were incubated with antibodies against $\beta$-catenin, E-cadherin, N-cadherin, cyclin D1, MMP7, Axin2, LaminB, or GAPDH (1:1000, Abcam, Cambridge, UK). Then, the membranes were incubated with a secondary antibody (Beyotime Institute of Biotechnology) for $2 \mathrm{~h}$. The bands were detected using the BeyoECL Plus kit (Beyotime).

\section{Statistical analyses}

The data were analyzed using GraphPad Prism 8 software. They are shown as the mean \pm SD. Significant differences were evaluated using the Student's $t$-test or one-way analysis of variance (ANOVA) with the Bonferroni post-hoc test. Statistical significance was set at $P<0.05$.

\section{Results}

\section{The elevation of CXCL14 and its prognostic implication} in OC

To investigate the dysregulation of CXCL14 expression in OC cells, we measured the mRNA and protein levels of CXCL14 in 50 pairs of OC tissues and nontumor ovarian tissues. As presented in Fig. 1A, the mRNA levels of CXCL14 were higher in OC specimens than in non-tumor ovarian tissues. Similarly, CXCL14 expression was significantly higher in OC $(84 \%, 42$ in 50 cases) relative to non-tumor ovarian tissues, which was demonstrated using immunohistochemical staining (Fig. 1B). Moreover, we did not find any difference in CXCL14 expression between the serous $(n=34)$ and mucinous $(n=12)$ subtypes. The Cancer Genome Atlas (TCGA) and the Genotype-Tissue Expression (GTEx) projects were used to investigate the expression of CXCL14 in OC cells using GEPIA (http://gepia.cancerpku.cn/) [19]. As illustrated in Fig. 1C, CXCL14 was substantially upregulated in OC cells $(n=426)$ as compared to that in normal tissues $(n=88)$. Next, an analysis of the clinical characteristics of patients with OC revealed that CXCL14 was associated with histological grade, $\mathrm{pT} / \mathrm{pN} / \mathrm{pM}$ status, and FIGO stage $(P<0.05$, Table 1). Remarkably, Kaplan-Meier survival analyses (http://kmplot.com/analysis/index.php?p=service\& cancer $=$ ovar) indicated that $\mathrm{OC}$ patients with higher CXCL14 expression exhibited a shorter OS (patients were divided into high- and low-group based on the mean value. Cutoff $=203$; Fig. 1D). Finally, CXCL14 expression in OC cells (A2780, OVCAR3, CAOV3, and SKOV3) was relatively higher than that in the normal cell line, HOSEpiC (Fig. 1E). These findings indicate that CXCL14 is upregulated in OC, with higher levels correlating to worse OS.

\section{Silencing of CXCL14 inhibits OC cell growth}

To illustrate the biological function of CXCL14 in OC, gain- and loss-of-function assays were performed through the introduction of CXCL14 into OC cell and knockdown of CXCL14 in OC cell (Fig. 2A and B). Although there was a slightly different silencing efficiency between sh-CXCL14 \#1 and sh-CXCL14 \#2 in OVCAR3 cell, the difference was not significant. However, shCXCL14 \#2 produced a more efficient silencing effect in SKOV3 cell. After comprehensive consideration, shCXCL14 \#2 was selected for functional studies. CXCL14 knockdown noticeably suppressed OC cell growth, as evidenced by CCK-8 (Fig. 2C) and colony formation assays (Fig. 2D). In contrast, ectopic overexpression of CXCL14 in OC cell yielded opposite results (Fig. 2E and F). Collectively, these findings suggest that CXCL14 serves as an oncogene and facilitates OC cell growth.

\section{Silencing of CXCL14 inhibits OC cell migration, invasion and EMT}

Next, we investigated the effect of CXCL14 on OC cell invasiveness and the EMT process. Firstly, the wound healing assay exhibited that CXL14 deletion restrained the migratory capacity of $\mathrm{OC}$ cell; however, upregulation 


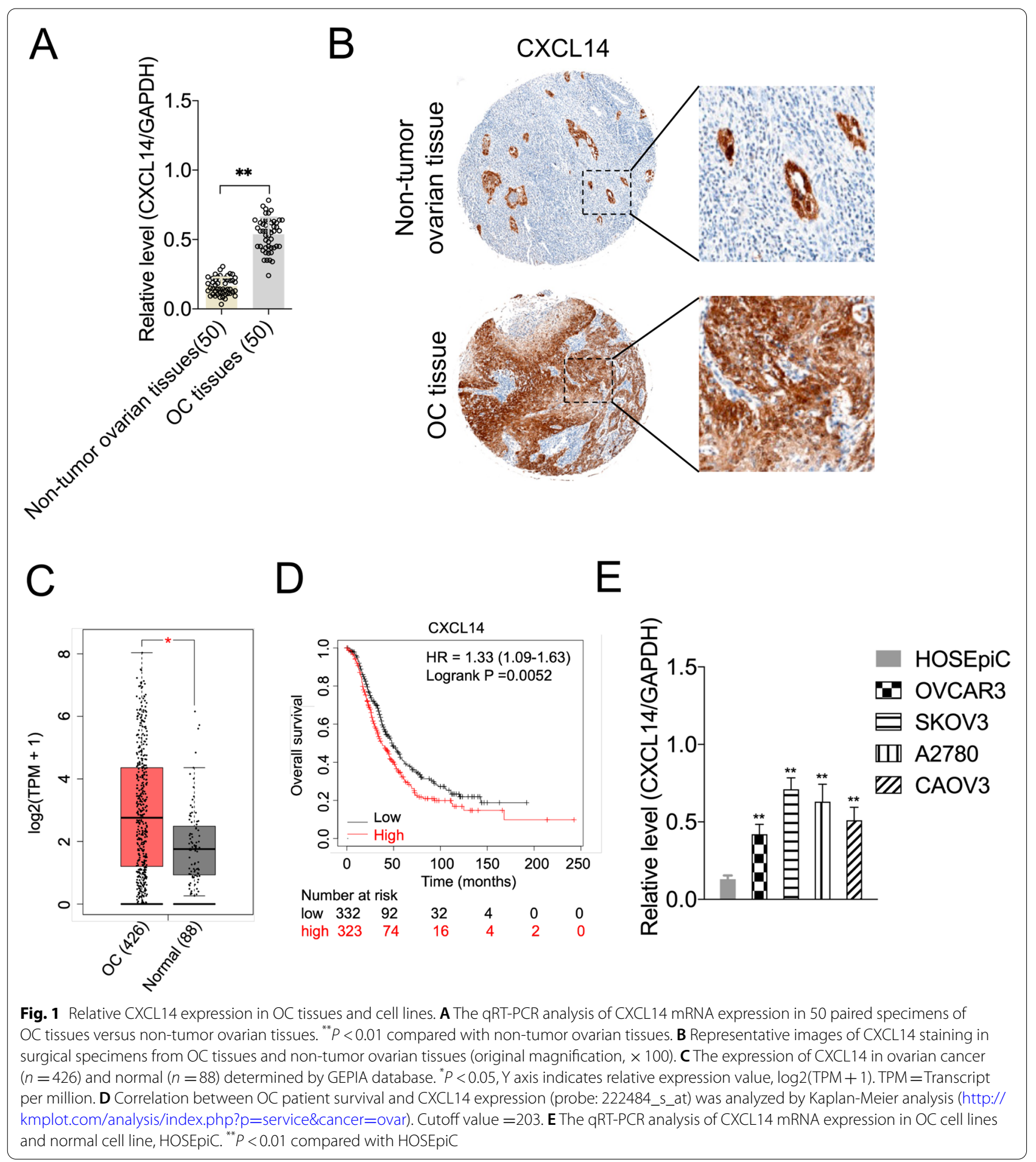

of CXL14 promoted cell migration (Fig. 3A and B). Transwell assay also revealed that CXCL14 knockdown suppressed the invasive ability of OC cell, whereas CXCL14 upregulation increased $\mathrm{OC}$ cell invasion (Fig. $3 \mathrm{C}$ and D). The expression of E-cadherin was increased in two cell lines after silencing of CXCL14, whereas N-cadherin expression was decreased (Fig. 3E). Nevertheless, the epithelial marker, E-cadherin, was downregulated in the OC cell after CXCL14 upregulation, and the protein expression of the mesenchymal marker $\mathrm{N}$-cadherin was 
Table 1 Association of CXCL14 expression with patient's clinicopathological features in ovarian carcinomas

\begin{tabular}{|c|c|c|c|c|}
\hline \multirow{2}{*}{$\begin{array}{l}\text { Clinical } \\
\text { parameter }\end{array}$} & \multicolumn{3}{|l|}{ CXCL14 } & \multirow[t]{2}{*}{$P$-value } \\
\hline & All cases & Low expression & $\begin{array}{l}\text { High } \\
\text { expression }\end{array}$ & \\
\hline Age (years) & & & & 0.414 \\
\hline$\leq 51$ & 16 & 7 & 9 & \\
\hline$>51$ & 34 & 20 & 14 & \\
\hline $\begin{array}{l}\text { Histological } \\
\text { type }\end{array}$ & & & & 0.348 \\
\hline Serous & 34 & 20 & 14 & \\
\hline Mucinous & 12 & 5 & 7 & \\
\hline Others $^{\mathrm{a}}$ & 4 & 2 & 2 & \\
\hline pT status & & & & 0.024 \\
\hline pT1 & 20 & 13 & 7 & \\
\hline pT2 & 13 & 8 & 5 & \\
\hline pT3 & 17 & 6 & 11 & \\
\hline pN status & & & & 0.001 \\
\hline pNO & 28 & 19 & 9 & \\
\hline pN1 & 22 & 8 & 14 & \\
\hline pM status & & & & 0.018 \\
\hline $\mathrm{pMX}$ & 34 & 22 & 12 & \\
\hline pM1 & 16 & 5 & 11 & \\
\hline FIGO stage & & & & 0.029 \\
\hline I & 9 & 7 & 2 & \\
\hline$\|$ & 13 & 8 & 5 & \\
\hline III & 18 & 8 & 10 & \\
\hline IV & 10 & 4 & 6 & \\
\hline
\end{tabular}

${ }^{*}$ Chi-square test

${ }^{a}$ Endometrioid, clear cell and undifferentiated types

FIGO International Federation of Gynecology and Obstetrics

increased (Fig. 3F). These data suggest that CXCL14 promotes the EMT and invasion of OC cell.

\section{Wnt/ $\beta$-catenin signaling is involved in CXCL14-mediated OC cell growth and invasiveness}

The Wnt/ $\beta$-catenin signaling axis is tightly regulated by $\beta$-catenin, which can trigger the transcriptional activation of several metastasis-related genes. To determine the clinical relevance of CXCL14- $\beta$-catenin signaling in $\mathrm{OC}$, we performed immunohistochemical staining for $\beta$-catenin in $\mathrm{OC}$ tissues. As shown in Fig. 4A, $\beta$-catenin expression was significantly higher in OC (72\%, 36 in 50 cases) relative to non-tumor ovarian tissues. Moreover, a positive correlation between $\beta$-catenin and CXCL14 protein levels (Fig. 4B, $P<0.01, \mathrm{r}^{2}=0.61$ ) was observed in these $\mathrm{OC}$ tissues. Nuclear accumulation of $\beta$-catenin is the central event in the activation of the Wnt/ $\beta$ catenin signaling pathway. Next, we investigated whether CXCL14 can regulate $\beta$-catenin nuclear accumulation in OC cells. As shown in Fig. 4C, overexpression of CXCL14 enhanced the nuclear $\beta$-catenin level in SKOV3 and OVCAR3 cell. Next, we detected the changes in Wnt/ $\beta$ catenin downstream genes in CXCL14 knockdown or overexpressing OC cell. CXCL14 knockdown reduced the mRNA and protein levels of $\beta$-catenin, MMP-7, cyclin D1, and Axin2 in OC cells. In contrast, CXCL14 overexpression upregulated the mRNA and protein levels of $\beta$-catenin, Axin2, MMP-7, and cyclin D1 in OC cell (Fig. 4D-G).

To determine whether Wnt/ $\beta$-catenin signaling was involved in CXCL14-induced OC cell metastasis, the specific inhibitor of Wnt/ $\beta$-catenin signaling, XAV939, was utilized. XAV939 $(10 \mu \mathrm{M})$ treatment decreased the expression levels of MMP-7, cyclin D1, and Axin2 in CXCL14 overexpressing OC cells (Fig. 5A) [20]. Additionally, XAV939 suppressed CXCL14-facilitated OC cell growth in the CCK-8 (Fig. 5B) and clonogenic assays (Fig. 5C). Similarly, XAV939 suppressed CXCL14-facilitated $\mathrm{OC}$ cell migration and invasion in the wound healing and transwell assay, respectively (Fig. 5D-E). Finally, we analyzed the correlation between CXCL14 and Wnt/ $\beta$-catenin target genes in OC using TCGA dataset. The result of Pearson's correlation analysis revealed that CXCL14 expression was positively correlated with Axin2, cyclin D1, and MMP-7 levels in OC tissues (Fig. 5F-H). These observations indicated that $\mathrm{Wnt} / \beta$-catenin signaling was responsible for CXCL14-induced OC cell growth and invasion.

\section{Discussion}

Emerging evidence has established that CXCL14 is important for cell growth, invasion, metastasis, and chemoresistance in multiple cancers [21]. Although it has been reported that upregulated CXCL14 expression promotes OC proliferation, little is known about its biological role in the various pathological processes of $\mathrm{OC}$, including EMT and invasion [14]. Herein, we demonstrated that the CXCL14 level was elevated in carcinoma tissues as compared with para-cancerous tissues. Importantly, high CXCL14 expression is associated with metastasis and poor prognosis. Next, our results indicated that CXCL14 knockdown suppressed OC cell growth, colony formation, and metastatic phenotypes, whereas CXCL14 overexpression produced the opposite results. Further analyses revealed that the canonical Wnt/ $\beta$-catenin signaling is required for CXCL14-mediated OC cell growth, migration and invasion.

Cancer metastasis is a complicated process, and cancer cells need to acquire metastatic characteristics, such as decreased adhesiveness and increased migration and invasive ability, to escape the confines of the primary tumor site and establish distant metastases [22]. In addition to generating cancer stem cells and resulting in 


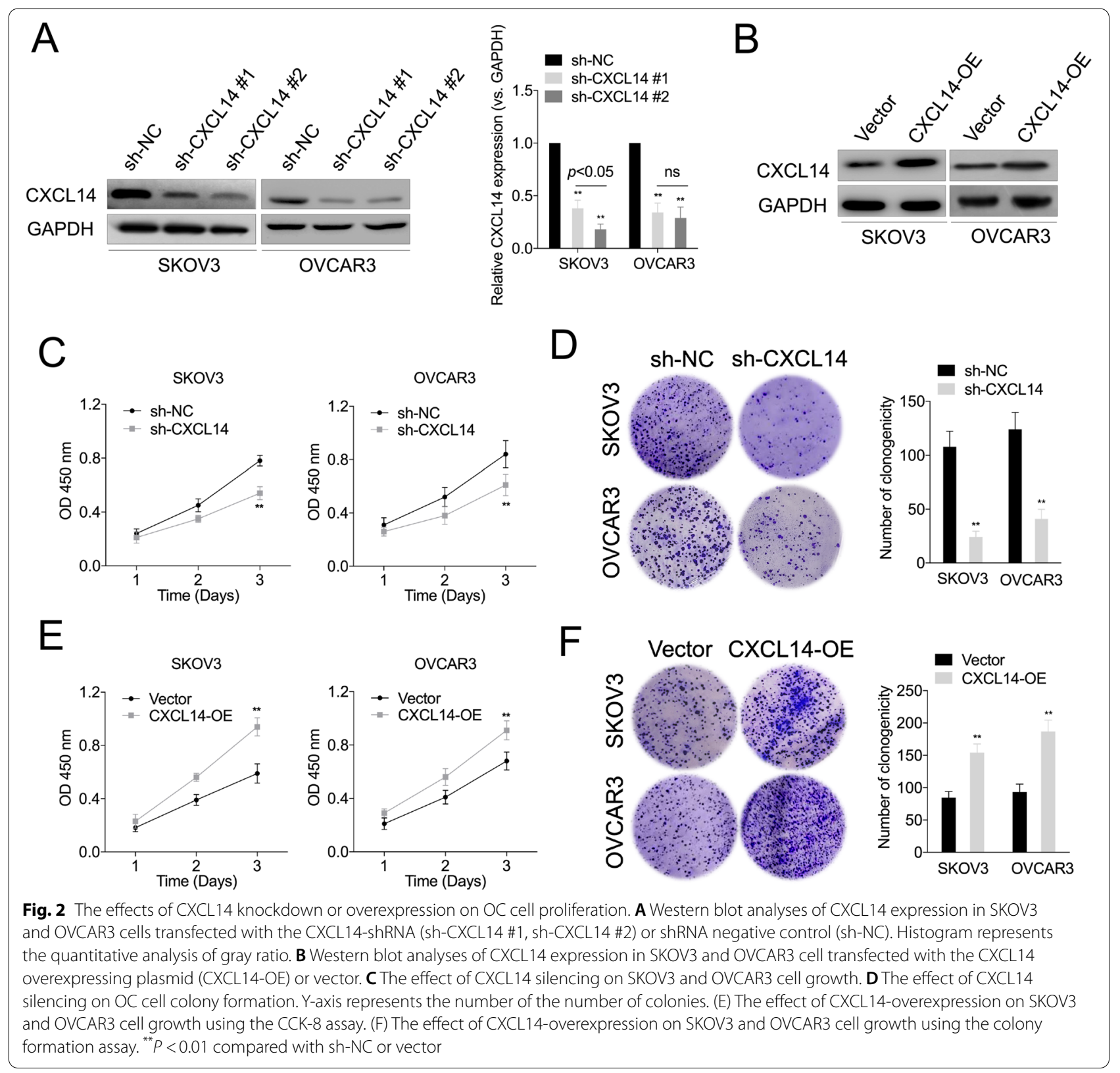

treatment resistance, EMT is considered to be necessary in the initial events of the metastasis cascade by conferring an aggressive phenotype [23]. Thus, the reversal of cancer cell EMT represents a considerable potential therapeutic option for patients with OC.

Importantly, Wnt $/ \beta$-catenin signaling was involved in the CXCL14-induced EMT process. First, the present findings revealed that reduced CXCL14 expression obstructed EMT, whereas upregulation of CXCL14 contributed to EMT, thus indicating that CXCL14 acts as a crucial regulatory factor of EMT in OC cells. Moreover, $\beta$-catenin is a vital element in the Wnt signaling pathway, and $\beta$-catenin facilitates the transcriptional activation of EMT-associated genes, including cyclin D1, MMP-7, and Axin2. Additionally, CXCL14 knockdown in SKOV3 cells lowered the expression of $\mathrm{Wnt} / \beta$-catenin-associated genes, whereas CXCL14 overexpression in OVCAR3 cells augmented their expression. To verify whether CXCL14 triggers OC cell growth and EMT via the Wnt/ $\beta$-catenin signaling pathway, OC cells were treated with XAV939. Intriguingly, inhibition of Wnt/ $\beta$-catenin signaling attenuated CXCL14-induced OC cell EMT, as well as cell growth and invasion. Thus, our data suggest that $\mathrm{Wnt} / \beta$-catenin signaling is necessary for CXCL14-facilitated OC cell growth and invasion. 

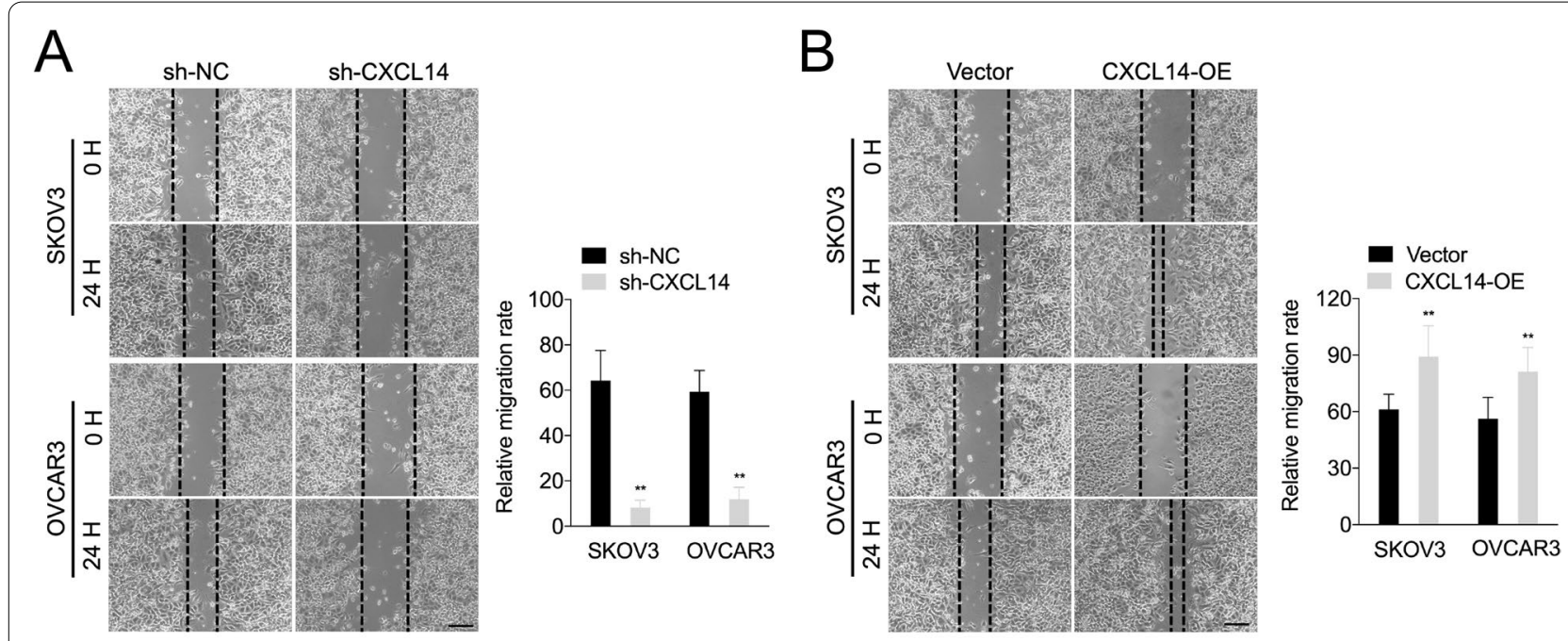

C
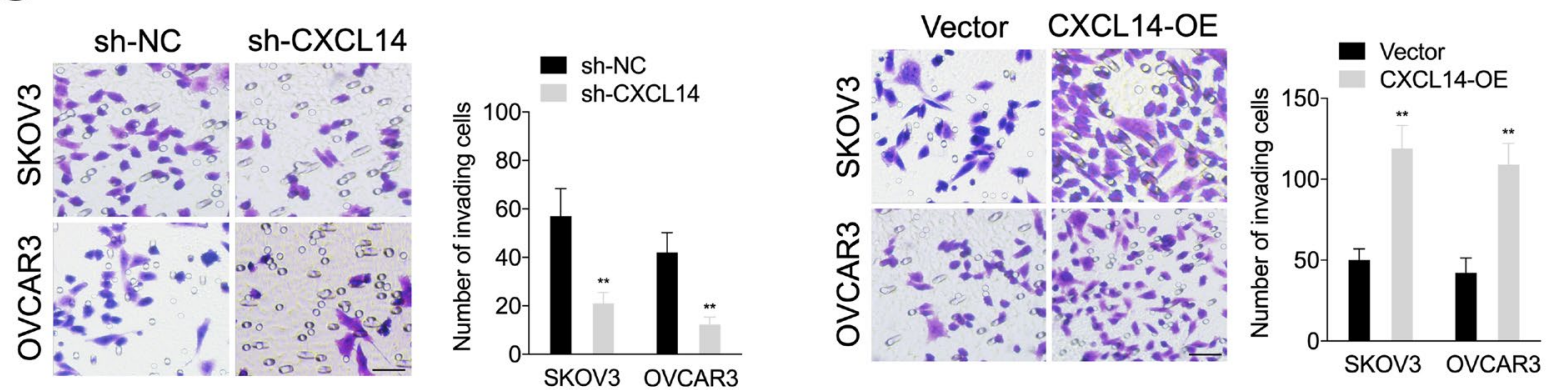

$E$
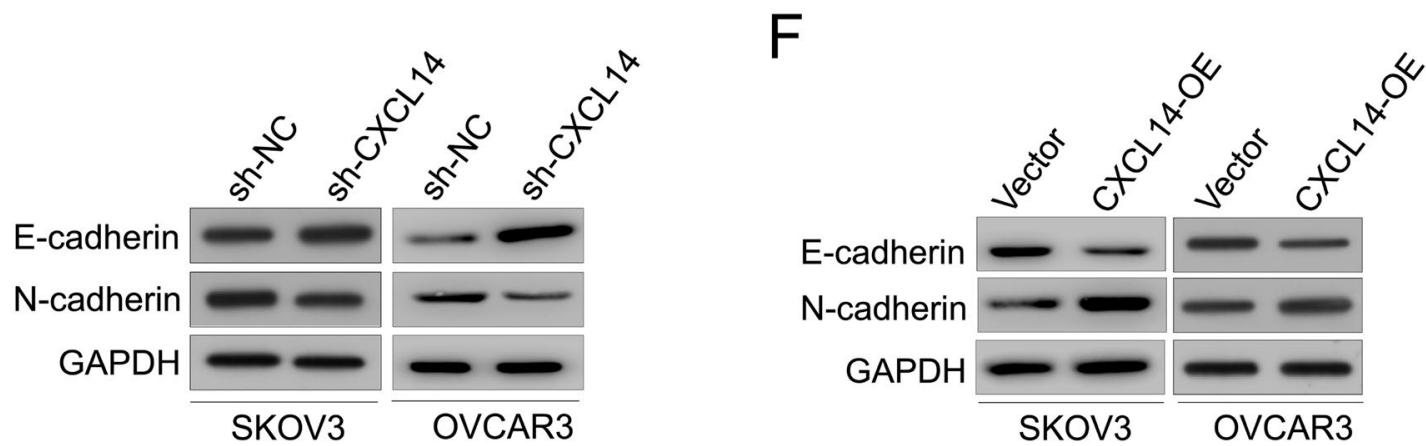

Fig. 3 The effects of CXCL14 on OC cell invasion and EMT. A-B The effects of CXCL14 downregulation or upregulation on the migration capabilities of OC cell using the wound healing assay. Scale bar: $200 \mu \mathrm{m}$. C-D The effects of CXCL14 downregulation or upregulation on the invasive capabilities of OC cells using the Transwell assay. Scale bar: $200 \mu \mathrm{m} .{ }^{* *} P<0.01$ compared with sh-NC or vector. E Western blot analyses of the expression levels of E-cadherin and N-cadherin in sh-CXCL14 transfected OC cell. F Western blot analyses of the expression levels of E-cadherin and N-cadherin in CXCL14 overexpressing OC cell

(See figure on next page.)

Fig. 4 The effects of CXCL14 knockdown or overexpression on Wnt/ß-catenin signaling-related markers. A Representative images of immunohistochemical staining of $\beta$-catenin in surgical specimens from OC tissues and non-tumor ovarian tissues (original magnification, $\times 100$ ). B Correlation study of CXCL14 and $\beta$-catenin in OC tissues. Pearson correlation coefficient were used for statistical analysis of the correlation between CXCL14 and $\beta$-catenin. CWestern blotting shows that overexpressing CXCL14 in OC cells increased the nuclear accumulation of $\beta$-catenin, respectively. ${ }^{* *} P<0.01$ compared with vector. D-E Following CXCL14 shRNA or overexpression treatment, qRT-PCR detection of the mRNA levels (cyclin D1, MMP-7, Axin2) in OC cells. ${ }^{* *} P<0.01$. F-G Following CXCL14 shRNA or overexpression treatment, western blotting detection of the protein expressions of cyclin D1, MMP-7 and Axin2 in OC cells. ${ }^{* *} P<0.01$ compared with sh-NC or vector 


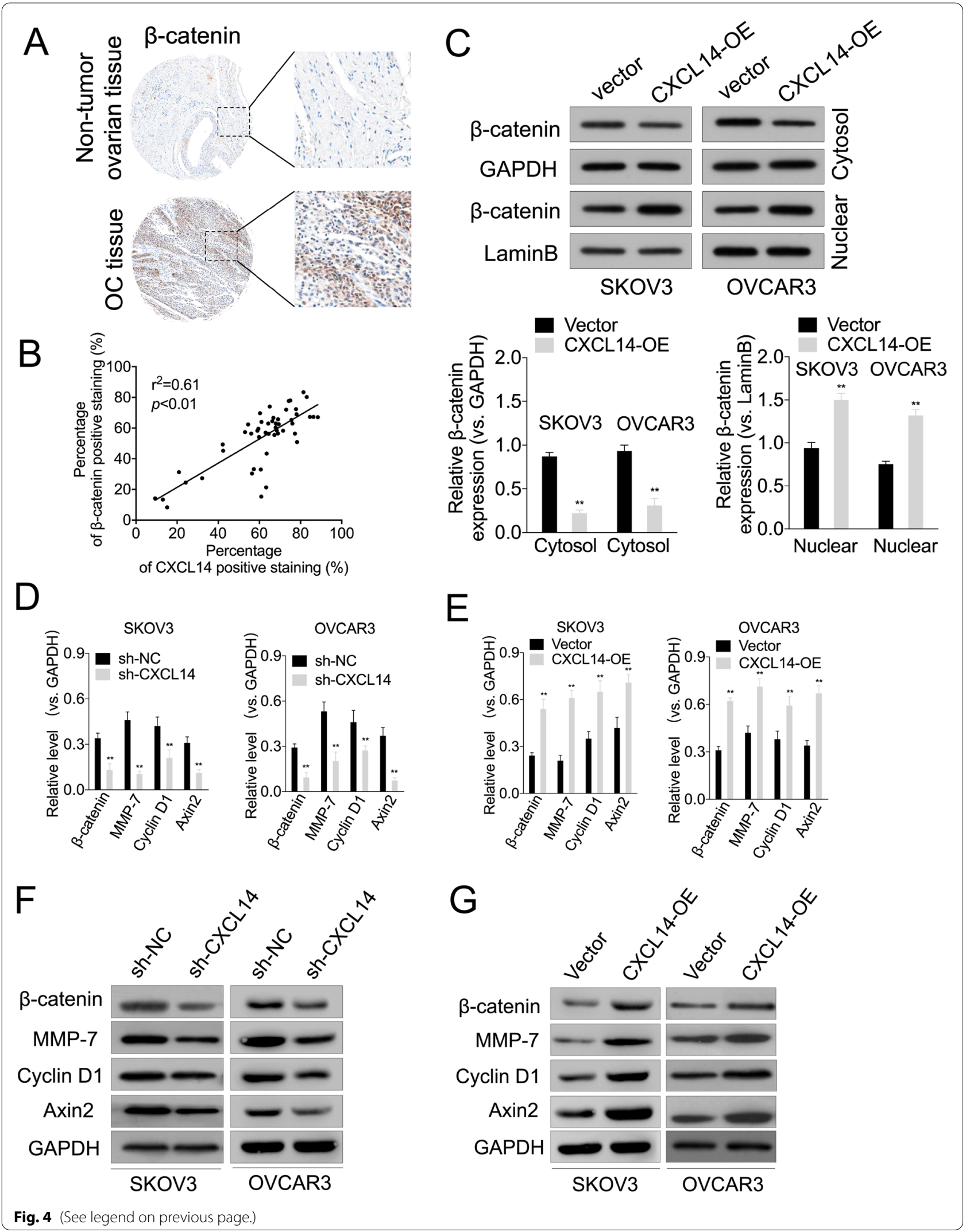




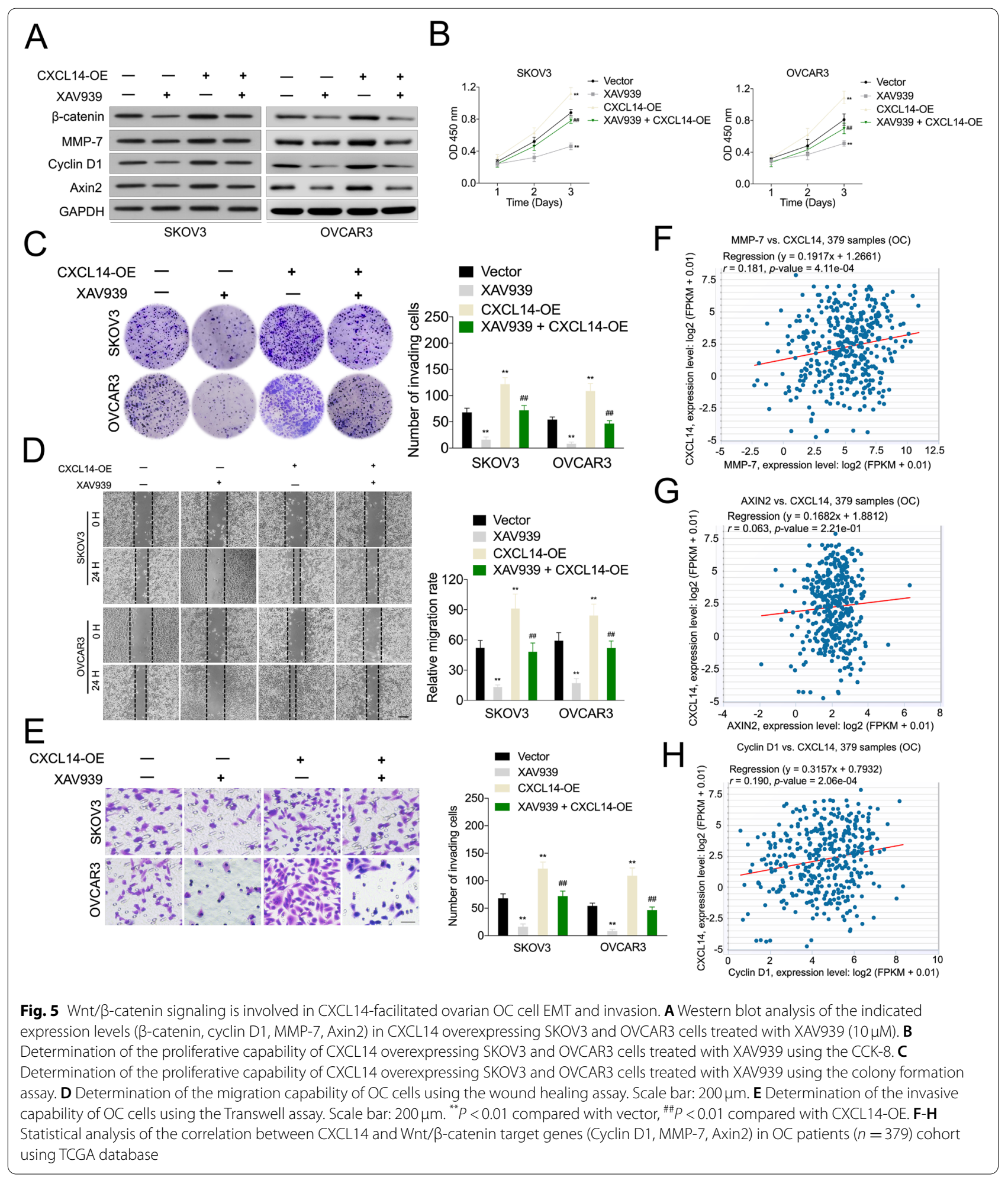

In tumor tissue cells, owing to various factors, the activation of signal transducer and activator of transcription 3 (STAT3) appears to be in a persistently and abnormally high expression state, which leads to abnormal proliferation of malignant tumor cells. Previous studies have shown that overexpression of CXCL14 increases the phosphorylation level of STAT3 in OC cells, thereby promoting the activation of the STAT3 


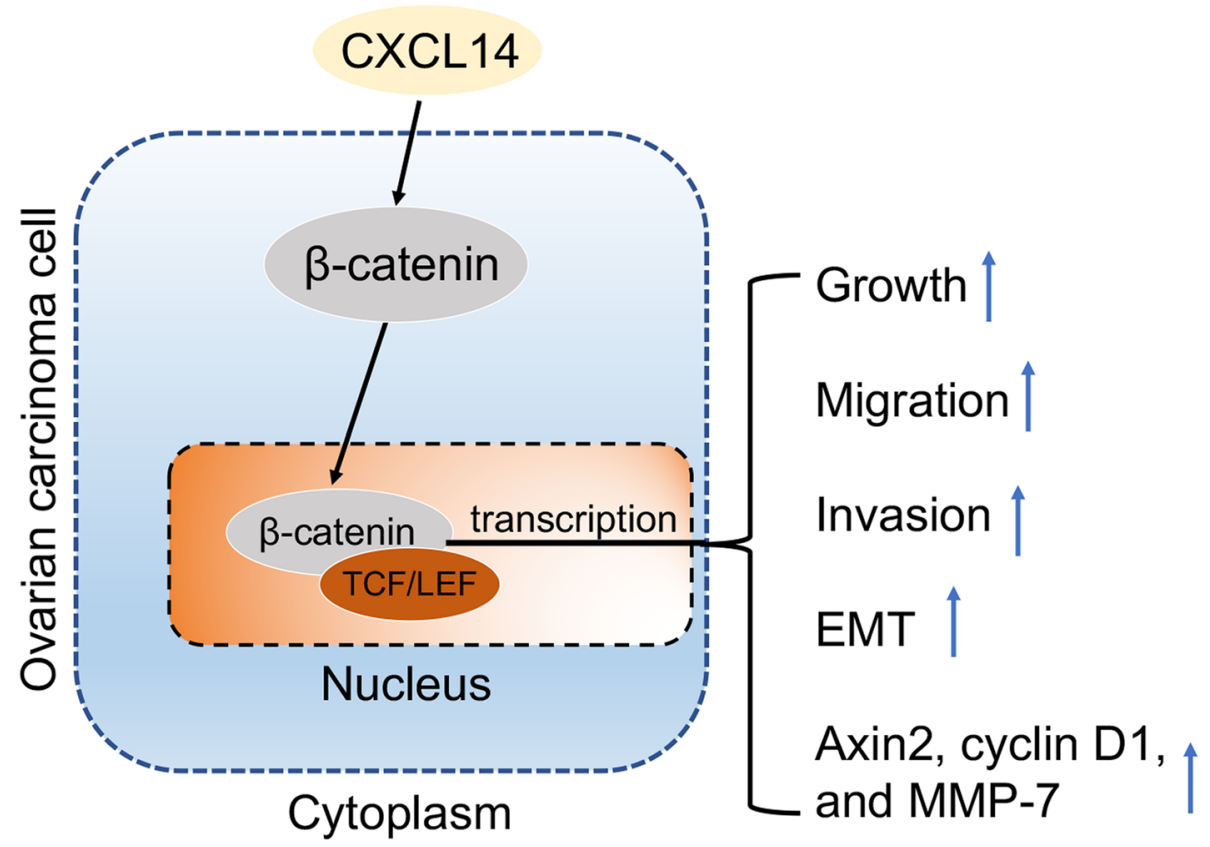

Fig. 6 Schematic diagram of the regulatory mechanism of the CXCL14/Wnt/ $\beta$-catenin signaling axis in promoting ovarian cancer cells migration, invasion and EMT

signaling pathway [14]. Moreover, it is indicated that STAT3 phosphorylation is involved in EMT and tumor metastasis. Specifically, several molecules have been demonstrated to promote tumor metastasis by activating the STAT3 signaling pathway [24, 25]. Accordingly, suppression of STAT3 phosphorylation downregulated the expression of mesenchymal markers ( $\mathrm{N}$-cadherin, Snail, and MMP-9) and increased the levels of the epithelial marker, E-cadherin [26]. However, additional investigations are required to identify whether STAT3 is involved in the CXCL14-facilitated OC cell invasion and EMT processes.

It is well recognized that nuclear $\beta$-catenin is associated with transcription factor 4 (TCF4) to activate target gene transcription [27]. The key molecular events in Wnt signaling are nuclear $\beta$-catenin accumulation and $\beta$-catenin/TCF4 complex transcriptional activity [28]. Nuclear $\beta$-catenin binds to members of the TCF/ LEF family of transcription factors, including Twist and Snail $1 / 2$, to trigger EMT $[29,30]$. Although $\beta$-catenin serves as a downstream effector in CXCL14-induced OC cell growth and invasion, it deserves further exploration of the complex interaction of $\beta$-catenin and CXCL14. To gain more insights into CXCL14-induced $\beta$-catenin transcriptional activity, the physical interaction between CXCL14 and $\beta$-catenin may be worth exploring in future studies. Furthermore, chemokines exert their effects by binding to specific transmembrane receptors. To date, several CXCL14 receptors have been identified, including insulin-like growth factor 1 receptor (IGF-1R) and C-X-C Motif Chemokine Receptor 4 (CXCR4) [13, 31]. Chemokine receptors have been reported to trigger the process of EMT and play an important role in cancer development [32, 33]. Further works are also needed to investigate which chemokine receptor plays the principal role in CXCL14-inducing OC cell EMT.

\section{Conclusion}

Altogether, our study demonstrates that high CXCL14 expression is associated with increased lymph node metastasis and worse prognosis in patients with OC. In conclusion, we found that CXCL14 promotes OC cell EMT and the metastatic phenotype by influencing the Wnt/ß-catenin signaling pathway (Fig. 6).

\section{Abbreviations \\ CXCL14: Chemokine (C-X-C motif) ligand 14; EMT: Epithelial-mesenchymal transition; OC: Ovarian cancer; aRT-PCR: Quantitative real-time PCR; CCK-8: Cell Counting Kit-8; TCGA: The Cancer Genome Atlas; STAT3: Signal transducer and activator of transcription 3.}

\section{Acknowledgements}

Not applicable.

\section{Authors' contributions}

Li-Na Gao, Man Hao, Xiao-Hui Liu, Li Zhang, Yan Dong and Yu-Fang Zhang conducted the research. Li-Na Gao, Man Hao and Xiao-Chun He analyzed the data and wrote the paper. All authors read and agreed with the final version of the paper. 


\section{Funding}

Not applicable.

\section{Availability of data and materials}

The datasets generated during the current study are available from the corresponding author on reasonable request.

\section{Declarations}

Ethics approval and consent to participate

The study was approved by the Medical Ethical Committee of Gansu Provincial Maternity and Child-care Hospital.

\section{Consent for publication}

Not applicable.

\section{Competing interests}

The authors declare that there is no conflict of interests regarding the publication of this paper.

Received: 17 June 2021 Accepted: 27 October 2021

Published online: 17 November 2021

\section{References}

1. Siegel RL, Miller KD, Jemal A. Cancer statistics, 2020. CA Cancer J Clin. 2020;70(1):7-30.

2. Bast RC Jr, Hennessy B, Mills GB. The biology of ovarian cancer: new opportunities for translation. Nat Rev Cancer. 2009;9(6):415-28.

3. Wu X, Zhao J, Ruan Y, Sun L, Xu C, Jiang H. Sialyltransferase ST3GAL1 promotes cell migration, invasion, and TGF-beta1-induced EMT and confers paclitaxel resistance in ovarian cancer. Cell Death Dis. 2018;9(11):1102.

4. Sun J, Yang X, Zhang R, Liu S, Gan X, Xi X, et al. GOLPH3 induces epithelialmesenchymal transition via Wnt/beta-catenin signaling pathway in epithelial ovarian cancer. Cancer Med. 2017;6(4):834-44.

5. Westrich JA, Vermeer DW, Silva A, Bonney S, Berger JN, Cicchini L, et al. CXCL14 suppresses human papillomavirus-associated head and neck cancer through antigen-specific CD8(+) T-cell responses by upregulating MHC-I expression. Oncogene. 2019;38(46):7166-80

6. Westrich JA, Vermeer DW, Colbert PL, Spanos WC, Pyeon D. The multifarious roles of the chemokine CXCL14 in cancer progression and immune responses. Mol Carcinog. 2020;59(7):794-806.

7. Kondo T, Ozawa S, Ikoma T, Yang XY, Kanamori K, Suzuki K, et al. Expression of the chemokine CXCL14 and cetuximab-dependent tumour suppression in head and neck squamous cell carcinoma. Oncogenesis. 2016;5(7):e240.

8. Lin K, Zou R, Lin F, Zheng S, Shen X, Xue X. Expression and effect of CXCL14 in colorectal carcinoma. Mol Med Rep. 2014;10(3):1561-8.

9. Gu XL, Ou ZL, Lin FJ, Yang XL, Luo JM, Shen ZZ, et al. Expression of CXCL14 and its anticancer role in breast cancer. Breast Cancer Res Treat. 2012;135(3):725-35.

10. Fazi B, Proserpio C, Galardi S, Annesi F, Cola M, Mangiola A, et al. The expression of the chemokine CXCL14 correlates with several aggressive aspects of Glioblastoma and promotes key properties of Glioblastoma cells. Int J Mol Sci. 2019;20(10):2496.

11. Morein D, Erlichman N, Ben-Baruch A. Beyond cell motility: the expanding roles of chemokines and their receptors in malignancy. Front Immunol. 2020;11:952.

12. Wang $Y$, Weng $X$, Wang $L$, Hao M, Li Y, Hou L, et al. HIC1 deletion promotes breast cancer progression by activating tumor cell/fibroblast crosstalk. J Clin Invest. 2018;128(12):5235-50.

13. Tanegashima K, Suzuki K, Nakayama Y, Tsuji K, Shigenaga A, Otaka A, et al. CXCL14 is a natural inhibitor of the CXCL12-CXCR4 signaling axis. FEBS Lett. 2013;587(12):1731-5.
14. Li X, Zhao L, Meng T. Upregulated CXCL14 is associated with poor survival outcomes and promotes ovarian cancer cells proliferation. Cell Biochem Funct. 2020;38(5):613-20.

15. Zhang W, Fei J, Yu S, Shen J, Zhu X, Sadhukhan A, et al. LINC01088 inhibits tumorigenesis of ovarian epithelial cells by targeting miR-24-1-5p. Sci Rep. 2018:8(1):2876.

16. Yang GP, He WP, Tan JF, Yang ZX, Fan RR, Ma NF, et al. Overexpression of SLC12A5 is associated with tumor progression and poor survival in ovarian carcinoma. Int J Gynecol Cancer. 2019;29(8):1280-4.

17. Burkhalter RJ, Westfall SD, Liu Y, Stack MS. Lysophosphatidic acid initiates epithelial to mesenchymal transition and induces beta-cateninmediated transcription in epithelial ovarian carcinoma. J Biol Chem. 2015;290(36):22143-54

18. Yang F, Xu J, Li H, Tan M, Xiong X, Sun Y. FBXW2 suppresses migration and invasion of lung cancer cells via promoting beta-catenin ubiquitylation and degradation. Nat Commun. 2019;10(1):1382.

19. Tang Z, Li C, Kang B, Gao G, Li C, Zhang Z. GEPIA: a web server for cancer and normal gene expression profiling and interactive analyses. Nucleic Acids Res. 2017:45(W1):W98-W102.

20. Sun J, Yang X, Zhang R, Liu S, Gan X, Xi X, et al. GOLPH3 induces epithelialmesenchymal transition via Wnt/ $\beta$-catenin signaling pathway in epithelial ovarian cancer. Cancer Med. 2017;6(4):834-44.

21. Yang XY, Ozawa S, Kato Y, Maehata Y, Izukuri K, Ikoma T, et al. C-X-C motif chemokine ligand 14 is a unique multifunctional regulator of tumor progression. Int J Mol Sci. 2019;20(8):1872.

22. Chaffer $\mathrm{CL}$, Weinberg RA. A perspective on cancer cell metastasis. Science. 2011;331(6024):1559-64.

23. Aiello NM, Kang Y. Context-dependent EMT programs in cancer metastasis. J Exp Med. 2019;216(5):1016-26.

24. Han Y, Guo W, Ren T, Huang Y, Wang S, Liu K, et al. Tumor-associated macrophages promote lung metastasis and induce epithelial-mesenchymal transition in osteosarcoma by activating the COX-2/STAT3 axis. Cancer Lett. 2019:440-441:116-25.

25. Rokavec M, Oner MG, Li H, Jackstadt R, Jiang L, Lodygin D, et al. IL-6R/ STAT3/miR-34a feedback loop promotes EMT-mediated colorectal cancer invasion and metastasis. J Clin Invest. 2014;124(4):1853-67.

26. Huang H, Han Y, Chen Z, Pan X, Yuan P, Zhao X, et al. ML264 inhibits osteosarcoma growth and metastasis via inhibition of JAK2/STAT3 and WNT/ beta-catenin signalling pathways. J Cell Mol Med. 2020;24(10):5652-64.

27. Teeuwssen M, Fodde R. Wnt signaling in ovarian cancer stemness, EMT, and therapy resistance. J Clin Med. 2019;8(10):1658.

28. Wu L, Zhou Z, Han S, Chen J, Liu Z, Zhang X, et al. PLAGL2 promotes epithelial-mesenchymal transition and mediates colorectal cancer metastasis via beta-catenin-dependent regulation of ZEB1. Br J Cancer. 2020;122(4):578-89.

29. Nguyen VHL, Hough R, Bernaudo S, Peng C. Wnt/beta-catenin signalling in ovarian cancer: insights into its hyperactivation and function in tumorigenesis. J Ovarian Res. 2019;12(1):122.

30. Gao H, Yin FF, Guan DX, Feng YX, Zheng QW, Wang X, et al. Liver cancer: WISP3 suppresses hepatocellular carcinoma progression by negative regulation of beta-catenin/TCF/LEF signalling. Cell Prolif. 2019;52(3):e12583.

31. Cheng CL, Yang SC, Lai CY, Wang CK, Chang CF, Lin CY, et al. CXCL14 maintains $\mathrm{hESC}$ self-renewal through binding to IGF-1R and activation of the IGF-1R pathway. Cells. 2020;9(7):1706.

32. Lin $Y, M a Q$, Li L, Wang H. The CXCL12-CXCR4 axis promotes migration, invasiveness, and EMT in human papillary thyroid carcinoma B-CPAP cells via NF-KB signaling. Biochem Cell Biol. 2018;96(5):619-26.

33. Yu X, Wang D, Wang X, Sun S, Zhang Y, Wang S, et al. CXCL12/CXCR4 promotes inflammation-driven colorectal cancer progression through activation of RhoA signaling by sponging miR-133a-3p. J Exp Clin Cancer Res. 2019;38(1):32.

\section{Publisher's Note}

Springer Nature remains neutral with regard to jurisdictional claims in published maps and institutional affiliations. 